\title{
MEMS Wind Direction Detection: From Design to Operation
}

\author{
Richard J. Adamec \\ David V. Thiel \\ School of MicroElectronic Engineering, Griffith University \\ Nathan, Brisbane, Qld, Australia \\ email r.adamec@griffith.edu.au, d.thiel@griffith.edu.au,p.tanner@griffith.edu.au
}

Philip Tanner

\begin{abstract}
A multi-axis hotwire anemometer fabricated with bulk micro-machining and thin film semiconductor technologies is presented. Extending the operation of a single axis anemometer into 2 axes allows direction sensing over the full $0^{\circ}-360^{\circ}$ while maintaining the original ability to measure flow velocity via the forced convection heat loss. Eliminating separate heating and sensing elements and combining both functions into single element pairs provides a means of lowering the temperature and power requirements and reducing size and fabrication complexity of the sensor compared to previously seen designs. CAD and modeling have aided in preparation of a suitable design to be fabricated in house using primarily photolithography and wet chemical etching. Successful wind tunnel trials have paved the way for field-testing and a commercially realizable sensor.
\end{abstract}

\section{Keywords}

MEMS, hot wire anemometer, anemometry, thermoresistor

\section{INTRODUCTION}

Directional solid-state hot wire anemometers typically utilize designs varying on a common theme of a thermal differential forming across the heated surface of the device due to the non-uniform convection cooling effects across the heating/sensing elements created by the incident airflow being heated as it passes over the surface.

In the case of a multi-axis based design, the differential response of the paired sensing elements follow a sine and cosine approximation of the incident airflow angle The combined thermal power loss of the elements is used for the velocity measurement. Determination of direction requires simultaneous solution of the differential paired signals with equations such as a modified sine function proving a suitable approximation. Design of this sensor has been aided by the use of the CoventorWare CAD modeling package designed specifically for mechanical, electrical, thermal and fluid modeling of MEMS structures and has provided an otherwise impossible visualization of the thermal effects and operation of the sensor.
The thermal isolation provided by reverse etch formed Silicon Nitride $\left(\mathrm{Si}_{3} \mathrm{~N}_{4}\right)$ membrane (Figure. 1), and allows sufficient heating $\left(>45^{\circ} \mathrm{C}\right.$ above ambient temperature) with an input power of $25 \mathrm{~mW}$. Measured temperature differentials of $>10^{\circ} \mathrm{C}$ between the sensor elements require only modest gain for interfacing to the host system and provide good accuracy (typically less than $4 \%$ error) over the full $0^{\circ}-360^{\circ}$ incident angle and $0.5 \mathrm{~m} / \mathrm{s}$ to $>25 \mathrm{~m} / \mathrm{s}$ air flow velocities.

Typically hot wire anemometers have used separate heating and sensing elements, however combining both functions into single element pairs provides a means of lowering the temperature and power requirements and reducing size and fabrication complexity of the sensor compared to previous designs [1][2]. Prototypes have been fabricated in house using primarily photolithography and wet chemical etching.

Steps toward fabrication by a commercial foundry are in motion to produce sufficient sensors suitable for field trials beginning late 2003 .

\section{DESIGN}

The mapping of the Gaussian like distribution of the thermal profile in thermocouple flow sensor arrays has been demonstrated as a practical method in directional anemometry [3]. The typically large number of sensing elements ( $5 \times 8$ polar array) required to analyze this thermal map may be substantially reduced while still maintaining sufficient directional sensitivity if a different method of solution is applied. Reducing the sensor to quadrants (figure 1) and analyzing and solving the sine - cosine type relationship existing between orthogonal quadrant pairs can achieve a substantial decrease in sensor complexity.

Simultaneously solving the directional relationship enables the sensor to operate at unknown air velocities unlike relying on the thermal image alone. The differential outputs from the element pairs can be expressed in terms of incident airflow angle $(\theta)$ as

$$
y_{1}=A \cdot \sin (\theta+c)+d
$$




$$
y_{2}=B \cdot \sin (\theta+e)+f
$$

where:

$$
\begin{aligned}
& \theta \text { - incident airflow angle } \\
& \mathrm{y}_{1}-\mathrm{N}-\mathrm{S} \text { differential signal } \\
& \mathrm{y}_{2}-\mathrm{E}-\mathrm{W} \text { differential signal } \\
& \text { A - differential amplitude of N-S pair } \\
& \mathrm{B} \text { - differential amplitude of E-W pair } \\
& \mathrm{c}-\text { angular offset of N-S pair } \\
& \mathrm{d}-\text { DC offset of N-S pair } \\
& \mathrm{e}-\text { angular offset of E-W pair } \\
& \mathrm{f}-\text { DC offset of E-W pair }
\end{aligned}
$$

By ensuring the angular offset is keep constant and equal for the element pairs and all other geometry is keep symmetrical, the amplitudes $A$ and $B$ of the two differential signals can be assumed equal therefore

$$
A=B \text { and } c=e
$$

with the resulting simultaneous solution of

$$
\theta=\arctan \left(\frac{y_{1}-d}{y_{2}-f}\right)-c
$$

For the above solution equation (3), extra work is required after evaluating the equation. The arctan function has a period of $\pi$, thus giving two possible angles separated by $\pi$. To determine which is the correct angle, the sign (positive or negative magnitude) of the East-West element pair is used, resulting in the final solution to equations (1) and (2) being

$$
\theta=\arctan \left(\frac{y_{1}-d}{y_{2}-f}\right)-c-\pi\left(1+\frac{y_{2}-f}{a b s\left(y_{2}-f\right)}\right)
$$

Correction equations to compensate for packaging irregularities and any periodic discontinuity can be applied in the form of either a polynomial or periodic post correction function.

\section{FABRICATION}

Traditional semiconductor fabrication techniques are used for the majority of the sensor fabrication. This begins with a $600 \mu \mathrm{m}$ silicon $\langle 100\rangle$ wafer is used for the sensor substrate. The physical wafer properties (ie. thickness etc.) are not critical as the sensor is not an active electronic device but rather is using the silicon as a physical supporting structure only. A $1.5 \mu \mathrm{m}$ PECVD silicon nitride layer is grown on both sides of the wafer for the purposes of forming the sensor membrane on the top surface and a patternable mask on the bottom surface. The thermoresistive nickel elements are deposited via sputtering and patterned with wet chemical etching to produce the $20 \mu \mathrm{m}$ wide, $100 \mathrm{~nm}$ thick tracks for each element. Nickel has been chosen for it relatively high Temperature Coefficient of Resistance, (TCR), and good resistance to deterioration in an exposed environment. The last fabrication process step is the patterning of the silicon nitride on the reverse side of the wafer to provide a window through which the silicon will be bulk etched to leave the $1.5 \mu \mathrm{m}$ silicon nitride membrane supporting the nickel elements forming the sensor membrane.

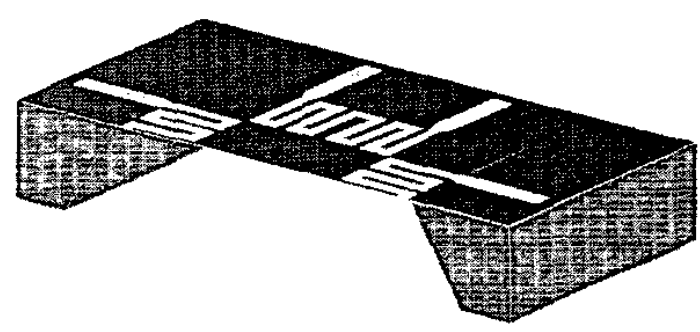

Fig 1. Reverse etching used to provide sufficient thermal isolation of the elements.

Thermocompression wire bonding from the element edges to a 16 pin 0.3 " ceramic IC carrier provides a means of connection of the sensor to the physical world.

As the sensor is to be exposed to the ambient environment protection of the device preferred. Protection from the environment must be limited to protecting the wire bonding as the sensor surface must be exposed to the environment which it is monitoring. An epoxy resin has been flowed around the sensor's circumference to provide both mechanical support/protection to the wire bonds with the combined purpose of providing a smooth, continuous surface from the edge of the sensor surface to the surface of the IC carrier. This smooth interface between the carrier and sensor is important to maintain laminar airflow over the sensor surface. Without this resin potting, the discontinuity at the sensor edge introduces turbulence that will degrade the sensor's performance. 


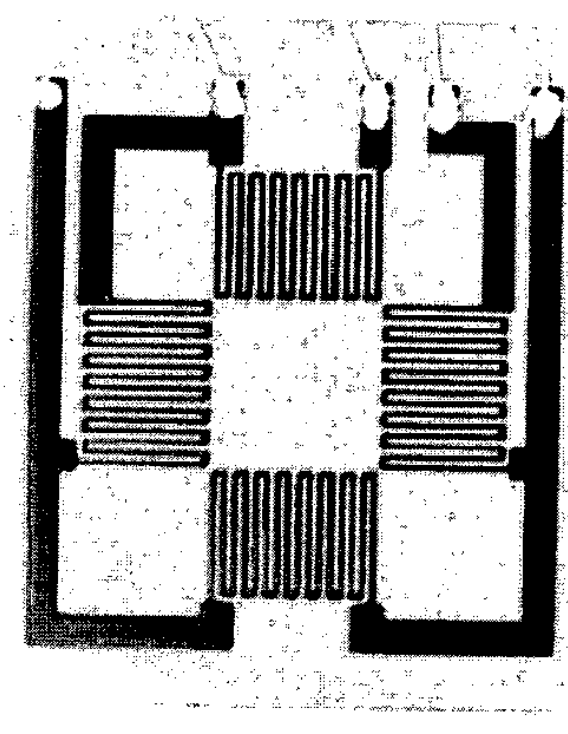

Figure 2. Optical micrograph of example sensor. $(2000 \mu \mathrm{m} \times 2000 \mu \mathrm{m})$

\section{OPERATION}

The operation of the directional anemometer has been investigated using three modes of operation; constant voltage, constant current, constant resistance.

Typical operation generates element temperatures of $35^{\circ} \mathrm{C}$ above ambient with a required power of $25 \mathrm{~mW}$ and achieving $>90 \%$ final element temperature within $100 \mathrm{~ms}$. Element temperatures in excess of $500^{\circ} \mathrm{C}$ have been reached with an input power of $250 \mathrm{~mW}$.

Current sources are commonly used for excitation of such devices and resistance feedback often not required due to low resistance variations during operation, however high power modes of operation can lead to instability and selfdestruction of positive temperature coefficient of resistance (PTCR) devices. Voltage or resistance feedback can provide stable operation due its self-limiting nature in a PTCR device. Temperature/resistance monitoring of the sensing elements provides a means to achieve stable "above ambient" temperatures of the heating elements and provides reduced sensitivity to fluctuations in ambient air temperatures. As the convection cooling of the sensor surface follows Newton's Law of Cooling, by maintaining a constant above ambient element temperature the only variable that may be effected is the convection cooling coefficient which will be primarily dependent on the air velocity. This results in a more linear and consistent response to the incident airflow.

\section{RESULTS}

Wind velocity measurements have shown the predicted sine / cosine relationship of the orthogonal sensor pair temperature differentials (figure 3 ). In solving these measurements with equation (4), the angle has been resolved to an accuracy of approximately $3 \%$.

After solution for the angle via equation (4) extra corrections may be applied. Either polynomial or periodic correction can be applied as the calculated angle can now be used in either the periodic domain over $0-2 \pi$ or over the finite range of $0^{\circ}-360^{\circ}$. With a $3^{\text {rd }}$ order polynomial correction, the error has been reduced to $<1.3 \%$, (figure 4 ).

Sources of error include airflow disruption due to the wire bonded connections standing proud of the sensor surface and the variation in airflow and direction due to the rectangular carrier package used and can be successfully compensated for with the correction methods.

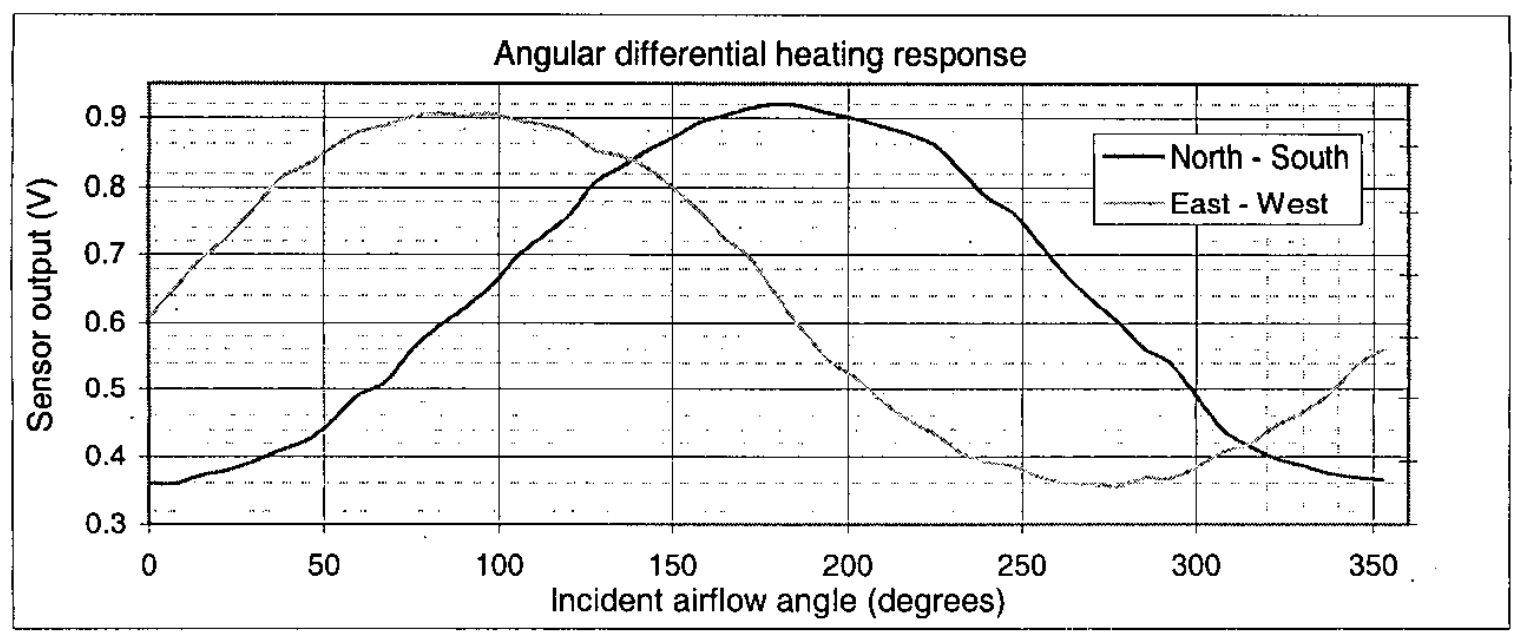

Figure 3. Differential heating response of each sensor pair. 


\section{SUMARY}

A MEMS based hot wire anemometer has been manufactured with primarily traditional thin film fabrication techniques and bulk silicon etching to complete the MEMS fabrication. Consideration in the geometrical design allows for a number of variations in the solution to be used and can provide advantages in the form of simplification of the solution for the directional measurement or flexibility with otherwise restrictive design constraints. The sensors default accuracy of $4 \%$ can be partially corrected with a number of correction methods and has been seen to be accurate to better than $1.3 \%$.

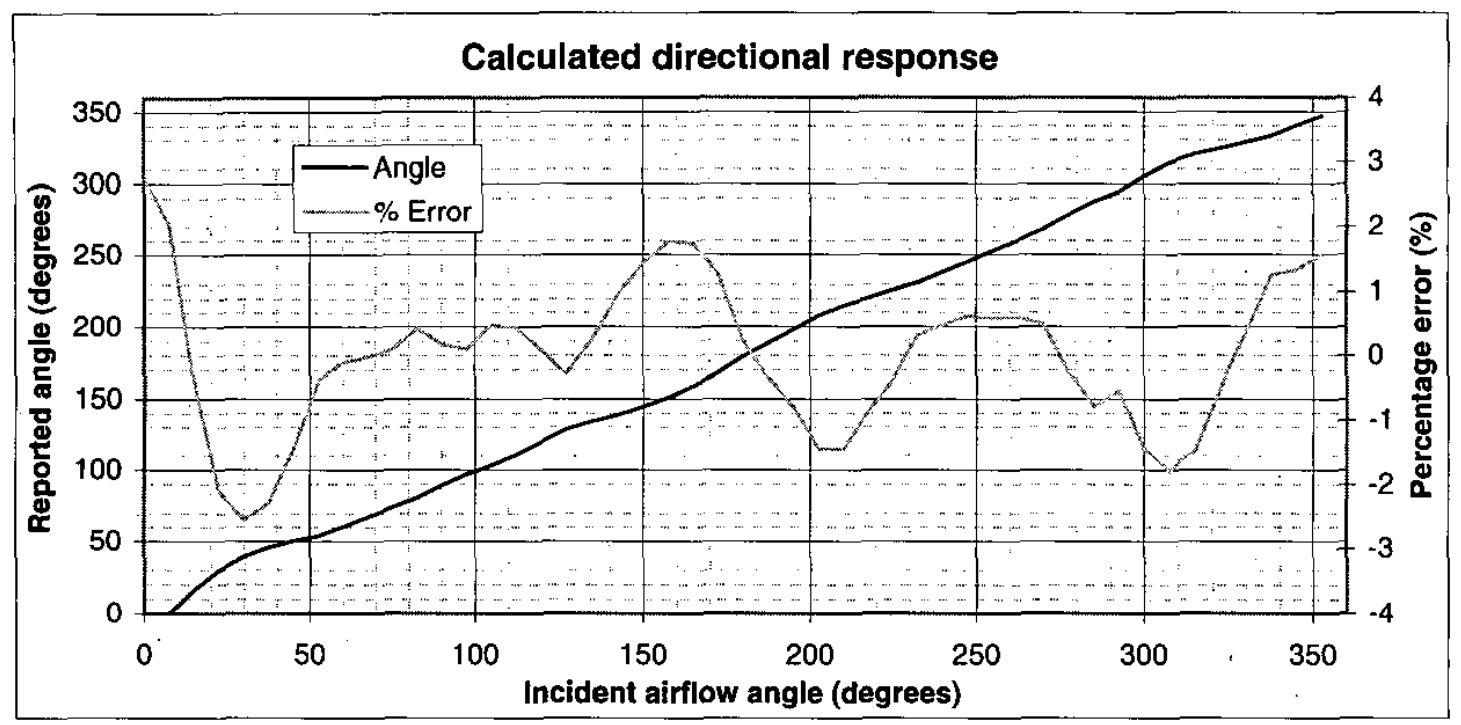

Figure 4. Calculated angle and associated error.

\section{REFERENCES}

[1] G. Bedo, H.Fannasch, R.Muller, "A silicon flow sensor for gases and liquids using $\mathrm{AC}$ measurements", Sensors and Actuators 852000

[2] K.A.A. Makinwa, J.H. Huijsing, "A wind-sensor interface using thermal sigma delta modulation techniques", Sensors and Actuators A 922001

[3] N. Nguyén, "A novel wind sensor concept based on thermal image measurement using a temperature sensor array", Eurosensors XVI, Sept. 2002 